\title{
Die Roboter kommen
}

VON HELMUT KREIDENWEIS

UND BERND HALFAR

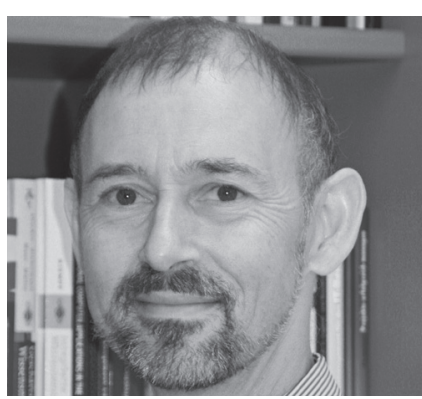

Prof. Helmut Kreidenweis ist Hochschullehrer für Sozialinformatik an der Katholischen Universität Eichstätt-Ingolstadt, Mitbegründer und Mitorganisator der ConSozial, Gründer und Vorstand des Fachverbandes Informationstechnologie in Sozialwirtschaft und Sozialverwaltung - FINSOZ e. V. sowie Beiratsmitglied der Zeitschriften Sozialwirtschaft und Sozialwirtschaft aktuell. E-Mail helmut.kreidenweis@ ku-eichstaett.de

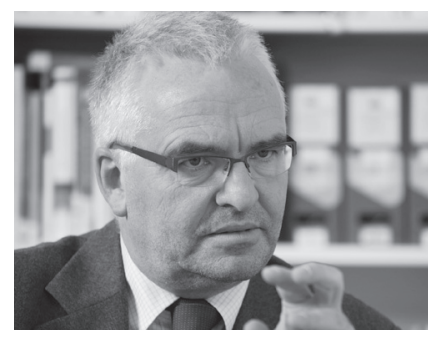

Prof. Dr. Bernd Halfar lehrt Management in sozialen Einrichtungen und Organisationsentwicklung an der Katholischen Universität Eichstätt-Ingolstadt. Er ist Mitgründer und Partner der Unternehmensberatung xit. forschen.planen.beraten $\mathrm{GmbH}$ mit Büros in Nürnberg und Berlin sowie Beiratsmitglied der Zeitschriften Sozialwirtschaft und Sozialwirtschaft aktuell. E-Mail bernd.halfar@ ku-eichstaett.de

\author{
Die Informationstechnologie verändert immer \\ stärker die Prozesse in Organisationen und \\ Unternehmen der Sozialwirtschaft. Zudem kündigt \\ sich eine weitere technische Durchdringung \\ sozialer Dienstleistungen an, von denen der \\ "Pflegeroboter« nur das populärste Beispiel ist. Nach \\ der Ökonomisierung der Sozialen Arbeit zeichnet \\ sich ihre Technisierung ab. Neben Kostenvorteilen \\ für die Leistungsanbieter sind dabei durchaus auch \\ Autonomiegewinne für die Nutzer vorstellbar.
}

Fachkräftemangel, Pflegenotstand, demografische Falle - Schlagwörter wie diese sind der Branche bestens vertraut. Eine mögliche Strategie, dieser künftigen Misere zu begegnen, ist es, menschliche Arbeitskraft durch technische Systeme (teilweise) zu ersetzen oder sie damit effizienter zu gestalten. Kaum gesagt schwebt schon das Schreckgespenst des Pflegeroboters über unseren Köpfen und raunt etwas von Entmenschlichung, sozialer Kälte und Arbeitsplatzvernichtung.

Beginnen wir mit der schnöden Computertechnik des beginnenden 21. Jahrhunderts: Die überwiegende Zahl der größeren Organisationen der Sozialwirtschaft hat sich längst ein ansehnliches Arsenal an Servern, Personal Computern und Breitbandnetzen zugelegt (vgl. Halfar/Kreidenweis, 2010). Intensiv nutzen sie Office, Internet, Mail \& Co - und der Sozialmanager lässt lässig sein neues iPad aus dem Lederetui gleiten. Auch bei aktuellen Technik-Themen wie Server- und Desktop-Virtualisierung sind zumindest die Abteilungen für Informationstechnologie (IT) vieler Komplexträger heute auf Augenhöhe mit der Industrie.

So weit so gut. Doch erst der Blick durchs Mikroskop zeigt die gefährlichen Erreger: Informationstechnologie ist akzeptiert, solange sie sich klaglos in die bisherigen Arbeitsgewohnheiten einpasst. Die Korrespondenz mit Word oder die Belegungsstatistik mit Excel sind sichtlich bequemer als Schreibmaschine und Taschenrechner, sie erfordern kein wirkliches Umdenken. Und ob der eigene Rechner tatsächlich auf dem Schreibtisch steht, oder nur noch virtuell in einer Server-Farm abgebildet ist, hindert niemanden daran, die liebevoll gebastelte Access-Datenbank weiter zu hätscheln.

Wenn sich Technik etwa in Form von Branchensoftware jedoch anschickt, Prozesse im Personalmanagement, der Dienstplanung oder der Erfassung und Weiterverarbeitung abrechnungsrelevanter Klientendaten mit zu prägen, ist schnell Feuer unterm Dach. Oder wenn der Wohnbereichsleitung gesagt wird, dass nun standardisierte Thin Clients die selbst geschnitzten Windows-Wildwüchse ersetzen sollen, ist der Aufstand ebenfalls vorprogrammiert.

In vielen Einrichtungen und Diensten wimmelt es nur so von Medienbrüchen, Mehrfacherfassungen, Wissenslücken und Software-Krücken. Gefühlte 


\section{Forschungsprojekt »easyCare«: Assistenz für pflegende Angehörige}

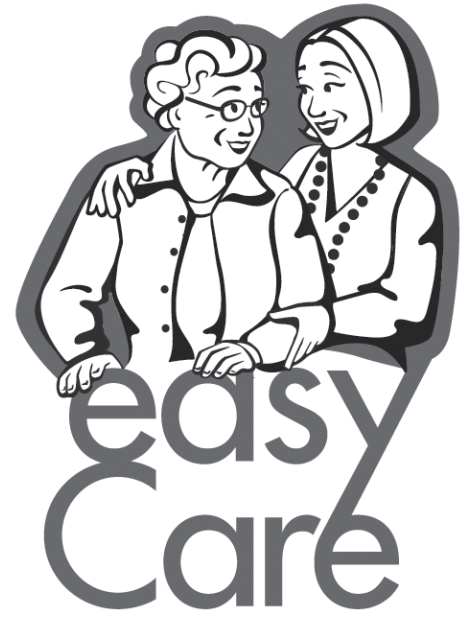

Das auf drei Jahre angelegte Verbundprojekt »easyCare« soll Angebote entwickeln, damit pflegebedürftige Menschen lange selbstbestimmt zu Hause leben können.

Das Vorhaben "easyCare" gehört zu den 18 Forschungsprojekten des Bundesministeriums für Bildung und Forschung, die seit 2008 unter dem Titel »Altersgerechte Assistenzsysteme für ein gesundes und unabhängiges Leben« mit einer Gesamtsumme von 45 Millionen Euro gefördert werden. Das Projekt »easyCare« ist Ende 2009 gestartet und sieht eine Laufzeit von drei Jahren vor. Insgesamt stehen dem Projekt 2,7 Millionen Euro zur Verfügung.

"easyCare" ist ein Verbundprojekt, das insgesamt vier Kooperationspartner aus
Wissenschaft, Forschung und Wirtschaft zusammenführt: das Forschungszentrum Informatik in Karlsruhe, das Wohlfahrtswerk für Baden-Württemberg in Stuttgart, die vitapublic $\mathrm{GmbH}$ in München und die Karlsruher RaumComputer $\mathrm{GmbH}$. Die Projektkoordination liegt in der Verantwortung des Forschungszentrums Informatik. Alle vier Partner garantieren mit ihren Kernkompetenzen und projektbezogenen Erfahrungen, das vornehmliche Ziel von "easyCare" zu erreichen: die gezielte Unterstützung von pflegenden Angehörigen, damit pflegebedürftige Menschen ein möglichst langes, selbstbestimmtes Leben in der gewohnten häuslichen Umgebung führen können.
Kern des Projekts ist ein zentrales Internetportal, das pflegenden Angehörigen mit den dringend benötigten Informationen und Dienstleistungen versorgt. Dazu gehören $u$. a. aktualisierte Pflegeinformationen, multimedial aufbereitete, leicht verständliche Pflegeschulungsinhalte und Social-Network-Komponenten sowie zielgruppengerechte Angebote von Pflegedienstleistern zur Pflegeunterstützung oder Beratung. Dazu gehört auch eine besondere Dienstleistung: Mit Case Management wird den pflegenden Angehörigen ein optimierter Gesamtversorgungsplan für den pflegebedürftigen Menschen zur Verfügung stehen, der einer ständigen

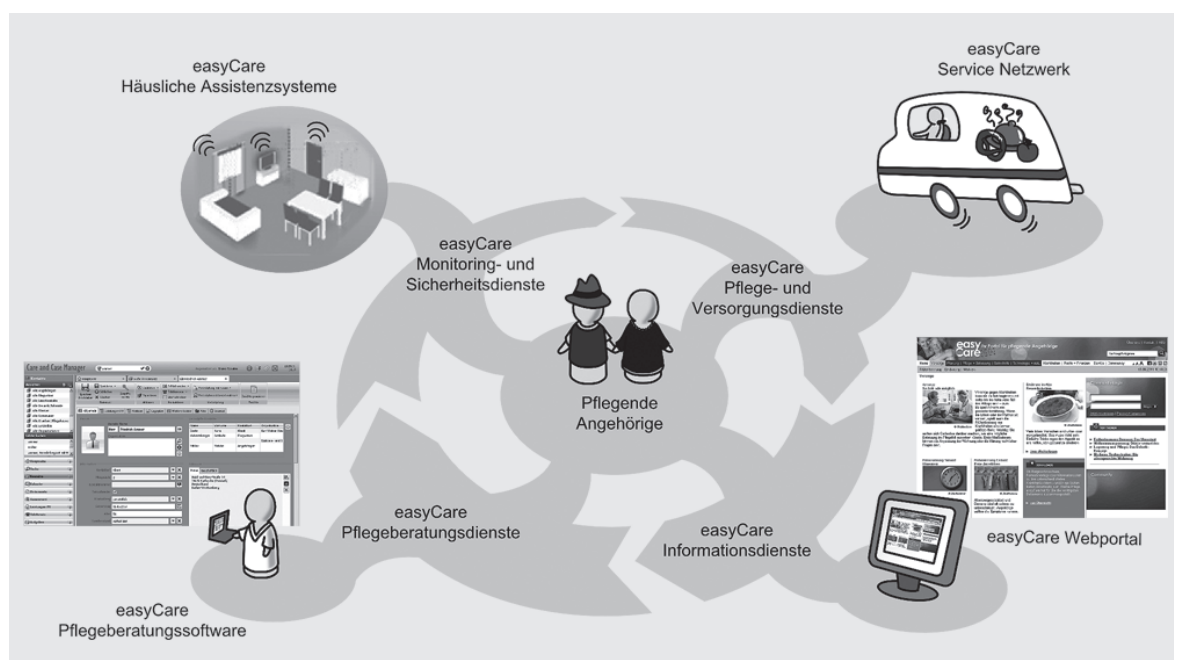

Im Verbundprojekt »easyCare« werden verschiedene Module für den Praxiseinsatz entwickelt.

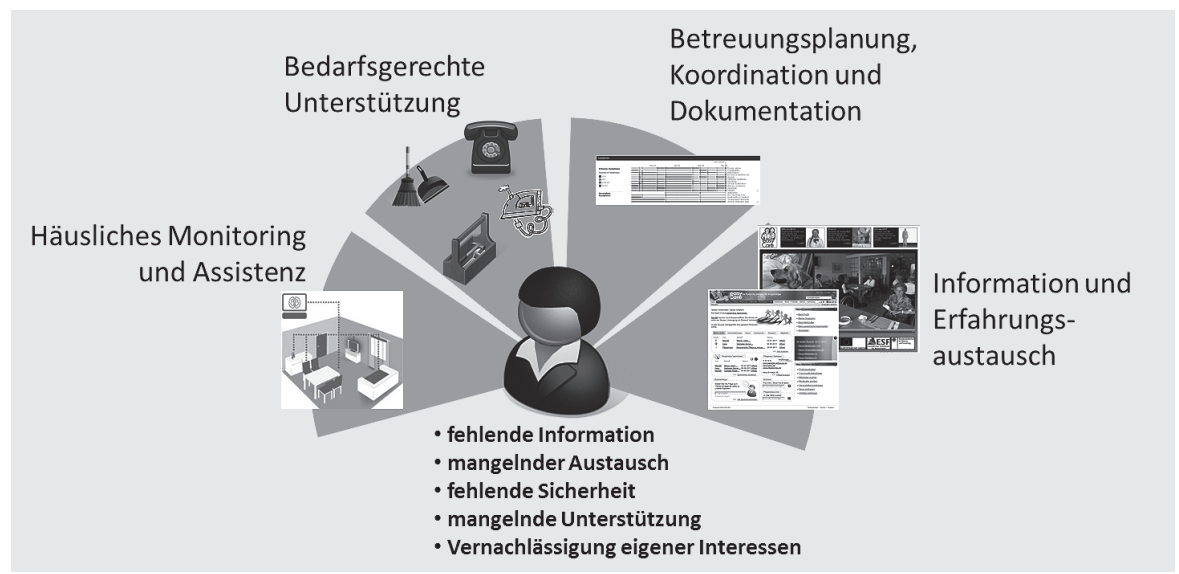

Die Unterstützung für pflegende Angehörige basiert im Projekt "easyCare« vor allem auf vier Säulen.
Kontrolle unterzogen wird. Insgesamt erhalten die pflegenden Angehörigen mit dem »easyCare«-Internetportal ein integratives Beratungs- und Betreuungsangebot, das deren Arbeit deutlich erleichtern wird.

Zum Erreichen der Projektziele sind acht Arbeitspakete definiert. Diese werden von mehreren Partnern bearbeitet, wobei sich für jedes Arbeitspaket jeweils ein Partner verantwortlich zeichnet. Dadurch wird eine höchstmögliche Transparenz in der Kooperation erreicht, um die Stärken der einzelnen Partner zielführend integrieren zu können.

FZI Forschungszentrum Informatik, Haidund Neustraße 10-14, 76131 Karlsruhe, Internet http://www.fzi.de
10 bis 20 Prozent Effizienzreserven schlummern hier zwischen akribisch abgehefteten Mails, in Word erfassten, ausgedruckten, gefaxten und neu eingegebenen Stammdaten oder selbst ge- strickten Excel-Dienstplänen mit kaum mehr durchschaubarem Formeldickicht.

Viele Führungskräfte haben die Informationstechnologie noch nicht als aktiv zu gestaltendes Management-The- ma auf ihrem Radarschirm - dort flackert sie nur hin und wieder als lästiger Kostenpunkt auf, wenn neue SoftwareLizenzen oder Server beschafft werden müssen. 
Doch der Wertschöpfungsbeitrag der Informationstechnologie und seine systematische Steigerung ist noch kein Thema, eher eine vage Hoffnung. Auf der anderen Seite finden sich nicht selten IT-Verantwortliche bei den Trägern, die in Gigabyte statt in Geschäftsprozessen denken und fleißig Server virtualisieren, auf denen dann ein bunter Flickenteppich an Anwendungsprogrammen lagert, der durch ungesteuerten Wildwuchs entstanden ist.

In den Spitzenverbänden gibt es für Qualitätsmanagement \& Co. tausende Arbeitskreise und Foren und Weiterbildungsangebot, doch Informationstechnologie kommt darin kaum vor. Die ITVerantwortlichen in den Einrichtungen sind meist isolierte Einzelkämpfer, institutionalisierte Kontakte zu Kollegen sind die Ausnahme. Kleines Beispiel: Das 380 Seiten starke Jahresprogramm 2012 der Bundesakademie für Kirche und Diakonie weist kein einziges Angebot zu diesem Themenfeld auf.

Dennoch - ein Umdenken deutet sich an. Bei der Frage, welche Ziele sie mit Informationstechnologie erreichen möchten, setzen Führungskräfte der Branche die Steigerung der fachlichen Qualität und die Effizienz von Arbeitsprozessen auf höchste Priorität - übrigens deutlich vor wirtschaftlichen Zielen wie Kostensenkung und Erlössteigerung. Doch die tatsächliche Erreichung dieser zentralen Ziele wird wesentlich geringer

\section{Fachverband als Dialogplattform}

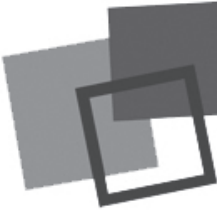

FINSOZ

FACHNERBAND

INFORMATIONSTECHNOLOGIE IN SOZIALWIRTSCHAFT

UND SOZIALVERWALTUNG E.V.

Im Jahr 2010 wurde der gemeinnützige Fachverband für IT in Sozialwirtschaft und Sozialverwaltung FINSOZ e. V. gegründet. Mitglieder sind vor allem große Träger aus allen Spitzenverbänden, Software-Anbieter für die Branche und wissenschaftliche Institute. Ziel des Verbandes ist es, den Nutzwert der Informationstechnologie in sozialen Organisationen zu steigern. Fortbildungen werden etwa zu Softwareauswahl, Usability oder IT-Compliance angeboten. Arbeitskreise beschäftigen sich mit der Professionalisierung des IT-Managements, mit Fragen des Datenaustauschs zwischen Leistungserbringern und Kostenträgern oder mit mobilen Lösungen.

Internet http://www.finsoz.de

Informationstechnologie ausgibt, wird bislang erstaunlicherweise kaum in Forschung zu Fragen wie diesen investiert. Wenig ist etwa über die maßgeblichen Faktoren für Qualitäts- und Effizienzeffekte bekannt, über deren methodische Messbarkeit und über deren Integration in die Dienstleistungsprozesse. So löst es etwa bestenfalls Achselzucken aus, wenn eine teure Dienstplan-Software eingeführt wurde, die die komplizierten Berechnungen von Zeitzuschlägen nach AVR oder TVöD automatisiert - die dadurch erzielten Effizienzgewinne aber in unbekannten Löchern versickern.

Immerhin: Mittlerweile gibt es bereits ein paar empirische Hinweise darauf,

\section{"Wenn die Technik liebgewordene Pro- zesse in der Organisation infrage stellt, ist schnell Feuer unterm Dach"}

eingeschätzt, zwischen Anspruch und Wirklichkeit klafft eine große Lücke (vgl. Halfar/Kreidenweis 2011).

Die Gretchenfrage lautet also: Wie muss die Computertechnik eingesetzt werden, dass die menschliche Arbeitskraft auf diejenigen Tätigkeiten konzentriert werden kann, die nicht durch Technik substituiert werden können oder sollen?

Obwohl die Sozialwirtschaft Jahr für Jahr rund eine Milliarde Euro für wo sich der Hebel sinnvoll ansetzen lässt: So zeigt etwa der IT-Report 2011 (a. a. O.), dass diejenigen Organisationen ihre IT-Ziele besser erreichen, die über eine schriftlich fixierte IT-Strategie verfügen und in denen Transparenz über IT-Kosten herrscht - beides nichts anderes als Indikatoren für ein professionelles IT-Management. Umgekehrt tun sich Einrichtungen schwer, die Potenziale der Informationstechnologie auszuschöpfen, wenn sich ihre Technik-
Perspektiven weitgehend auf Druckerpatronenpflege und Speichererweiterung beschränken.

Andere Studien (etwa Kreidenweis, 2009) zeigen, dass die Passgenauigkeit von fachlichem Konzept und Programmfunktionalität, gut getakteter Schulung und professionell organisierter Anwenderbetreuung wesentliche Erfolgsfaktoren darstellen - kurz: Es muss kompetente Kümmerer für Einführung und Betrieb der Programme geben.

Eine jüngst durchgeführte qualitative Befragung von pädagogischen Fachkräften eines Komplexträgers (vgl. Dilger/ Kreidenweis 2012) konnte zeigen, dass entgegen manchen Vermutungen die Akzeptanz der Computertechnik als solche heute nicht mehr das große Thema ist. Vielmehr wird die Technik akzeptiert, aber in typischen Deutungsmustern. So verknüpfen Fachkräfte die Informationstechnologie häufig eng mit Kontrolle durch Vorgesetzte oder mit wachsenden Dokumentationspflichten, deren Ursache in der Informationstechnologie gesehen wird.

Neue Hinweise liefert auch eine aktuelle Studie, die sich mit Methoden der Qualitätsmessung von Fachsoftware beschäftigt. Am Beispiel unterschiedlicher Pflegedokumentationsprogramme wird die Anzahl der notwendigen Mausklicks ermittelt, die erforderlich sind, um eine pflegerische Maßnahme zu terminieren (vgl. Koukouliatas in diesem Heft).

An dieser Stelle sei ein kurzer Blick auf den aktuellen Software-Anbietermarkt gestattet: Noch immer tummeln sich in Deutschland mehrere hundert Firmen zwischen Kindertagsstätte und 


\section{Ausblick: So kann es gehen}

Die Technik kann helfen, mit der »Partizipation des Betroffenen« Ernst zu machen:

- Der fußballvernarrte Mensch mit geistiger Behinderung wird auf dem Touchscreen das Gesicht seines Lieblingsbetreuers antippen und ihn für einen Stadionbesuch am nächsten Samstag buchen. Diese elektronische Buchung übernimmt das Dienstplanprogramm.

- Die Jugendlichen in der Trabantenstadt doodeln sich selbst die Öffnungszeiten des offenen Jugendtreffs zusammen.

- Der Professor findet in der Suchtdatenbank statistische Zwillinge und entsprechend konfigurierte Trainingsprogramme im Netz.

- Das Lese-Rechtschreib-Schwäche-Nintendospiel mit integriertem Aggressionshemmer liegt unter den Christbäumen.

- Der blinde Sachbearbeiter fährt via Induktionsschleife im eigenen Auto nach Hause.

- Im Bereitschaftszimmer des Altenpflegeheimes werden über Bildschirme und Sensortechnik 80 Bewohner während der Nacht überwacht, von denen die Hälfte durch dieses Monitoring in der eigenen Wohnung wohnen kann.

- Die Jugendlichen in den Berufsbildungswerken verfolgen ihre Fortschritte in einer Fachsoftware und visualisieren diese in einem schicken Chart.

- Demenzkranke Menschen genießen die Bewegungsfreiheit, die Ihnen ein RFIDChip am Armband ermöglicht.

Und nicht zu vergessen: Die Aktenführung beim Medizinischen Dienst der Krankenkassen, die Hilfeplanakten im Jugendamt und die Erfassungsbögen zur Integrierten Teilhabeplanung der sozialpsychiatrischen Einrichtung gewinnen an Präzision und Qualität, seitdem die Klienten elektronischen Aktenzugang haben. Die Sozialarbeiter freuen sich über Korrekturen und Anmerkungen der Klienten.

Helmut Kreidenweis/Bernd Halfar

Altenheim, die meisten davon viel $\mathrm{zu}$ klein, um mit den rasanten Entwicklungen in der Informationstechnologie mitzuhalten, geschweige denn, in Kooperation mit Wissenschaft und guter Praxis innovative Impulse zu setzen.

Eine Marktbereinigung wäre heilsam, doch nur der Käufermarkt kann sie befördern. Wenn dort jedoch über weite Strecken das Wissen fehlt, um gute von schlechter Software zu unterscheiden, wird sich auch weiterhin das Programm mit sprödem 1990er-JahreCharme und 1980er-Jahre-Technologie unter der Haube verkaufen lassen. Verwandte Märkte, wie etwa der für Kliniksoftware, zeigen hier bereits einen deutlich höheren Reifegrad, der auch der Sozialwirtschaft zu wünschen ist.

Typische Schwachstellen gängiger Branchensoftware für die Sozialwirtschaft sind mangelnde Anpassungsfähigkeit und geringe Prozessorientierung. Bei den meisten Programmen drängt sich auch der Eindruck auf, dass das Internet noch seiner Erfindung harrt. Sozial-Software, die im Browser abläuft oder interaktive Web-Schnittstellen etwa für das Belegungsmanagement oder für Terminanfragen von Klienten muss man mit der Lupe suchen.
Ansonsten gibt es überall dort ausgereifte Lösungen, wo bundesweit vorgegebene oder zumindest in der Fachwelt anerkannte Standards für die Planung, Dokumentation und Abrechnung von Leistungen existieren. Dort wo man wie etwa in der Jugendhilfe - föderalen Wildwuchs und fachliche Flickenteppiche vorfindet, ist die Software-Nutzung schwierig und das Programm-Angebot eher dürftig.

Nicht nur die IT-Durchdringung sozialer Dienstleistungen wird künftig steigen, auch die Dienstleistungen selbst werden technischer werden. Mit der zunehmenden Vermischung von personaler Dienstleistung und technischen Artefakten sind zwei wesentliche Konsequenzen verbunden: Zum einen wird die »hybride soziale Dienstleistung « Kostenvorteile mit sich bringen, weil der »Pflegeroboter « zumindest keinen Überstundenausgleich, Weihnachtsgeld oder Supervision fordert, ja außer Strom und gelegentlich einer neuen Festplatte keine Bedürfnisse hat - und wenn Burn-out, dann im wahrsten Sinne des Wortes und auf Kosten der Haftpflichtversicherung.

Bedeutsamer als dieser direkte Kosteneffekt ist jedoch eine andere Konsequenz: Wir erwarten einen Umbruch in der Dienstleistungsgestaltung selbst. Nach der »Ökonomisierung « der Sozialen Arbeit kommt ihre »Technisierung «.

Technisierung von Dienstleistungen, ob Geldabheben am Bankautomat, Tanken an der elektronischen Zapfsäule, Kaffeeauswahl am Automat im Büro, Blutdruckmessen zu Hause, Insulinspritzen beim Meeting bedeutet auf der einen Seite (fast) immer, dass die Anteile an der Wertschöpfung der Dienstleistung, die durch den Klienten erbracht werden, steigen. Das ist nicht nur kosteneffizient, den Kunden weiter in die Dienstleistungserstellung zu integrieren, seine Eigenproduktionsanteile zu erhöhen, sondern verbessert auch die Autonomie des Klienten.

Mögliche Formen der Klientenintegration in Dienstleistungsprozesse (vgl. Kasten "Ausblick: So kann es gehen «) sind methodisch durch Service-Blueprintings abgesichert und niemand erinnert sich an die Zeiten, als behinderte Menschen ihre gewünschte Dienstleistungspalette nicht über Evidence-basedService-Konfiguratoren im Netz vornehmen konnten. Es war hilfreich, die Technologie der Car-Configurators auf die Behindertenhilfe zu übertragen.

Ein Meilenstein war die Übersetzung des Gesetzes für Rehabilitation und Teilhabe behinderter Menschen (SGB IX) in »einfache Sprache", um auch Gesetze barrierefrei zu gestalten. Wikipedia hat sich entschlossen, die Einträge in verschiedenen Schwierigkeitsklassen anzubieten. Die Städte atmen auf, seitdem die Erlöse aus den Immobilienverkäufen aller Jugend- und Sozialämter in der Kämmerei eingegangen sind. Die Sozialarbeiter sind von Montag bis Donnerstag ausschließlich bei den Klienten, die Aktenführung läuft über Tablet-PCs, Spracherkennungssoftware und eine Private Cloud. Der Behindertenfahrdienst hat seine Vorhaltekosten und Personalkosten gedrittelt. Die Fahrer sitzen in ihrem Wohnzimmer und steuern die Busse über Web und Satellit, es gibt keine Leerfahrten mehr und keine wartenden Fahrer. - Diese Beispiele klingen vielleicht etwas irre, sind aber coole Realität, in den Entwicklungsabteilungen und Laboren.

Technische Sozialinformatik-Lösungen, auch vergleichsweise banale Dinge wie Kameramonitoring, Trittsensoren, sich selbst abschaltende Küchenherde, Skyping oder elektronische Klienten- 
akten liefern Material für jede Menge Ambient Assisted Living (AAL)-Tagungen, AAL-Forschungsprogramme, AAL-Messestände und AAL-Drittmittelspezialisten - aber es gibt bis heute kaum real genutzte AAL-Anwendungen. Dieser Widerspruch zeigt ein Dilemma: Die sozialen Träger sind im AAL-Sektor nicht investitionsbereit, weil den Entscheidern offensichtlich nicht klar ist, wie man technisch beeinflusste Dienstleistungskonfigurationen bildet und ob sie sich wirtschaftlich lohnen.

\section{Resümee: Der Markt spaltet sich}

Offensichtlich steckt die Sozialinformatik in ihren klientenbezogenen Praxislösungen in einem »cultural lag«: Man kann sich moderne technisierte Dienstleistungen denken, aber nicht umsetzen. Eine Ausnahme bildet das bundesweit wahrscheinlich innovativste Sozialunternehmen Sozialwerk St. Georg in Gelsenkirchen, das AAL-Technologie im Wohnbereich bereits einsetzt.

Automatisierung der Dienstleistungen und Integration des Klienten in die Dienstleistungserstellung bilden die zwei Haupttrends bei der Technisierung der Sozialarbeit und Pflege. Und die Vermutung des ehemaligen IBM-Managers Gunther Dueck auf dem letzten Kongress der Sozialwirtschaft trifft wahrscheinlich auch auf den Sozialbereich zu: Die personalen Dienstleistungen in der Sozialarbeit werden stärker differenziert zwischen kreativer Expertenarbeit und routinierten Unterstützungsarbeiten.

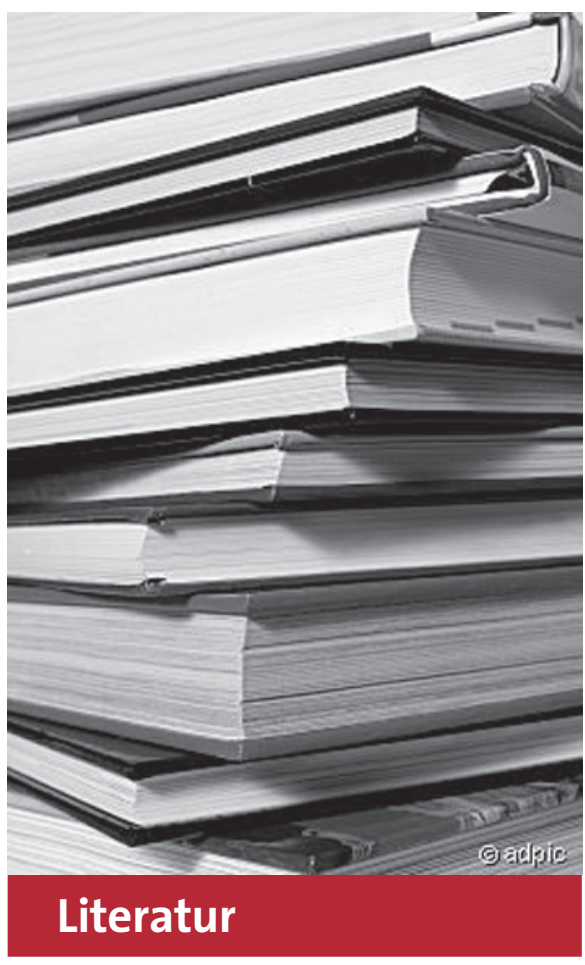

Dilger, Corvin/Kreidenweis, Helmut: IT von unten. Eine Rekonstruktion des FachkräfteBlicks auf Informationstechnologie in sozialen Organisationen. In Sozialmagazin 1/2012.

Dueck, Gunther: Aufbrechen! Warum wir eine Exzellenzgesellschaft werden müssen. Frankfurt am Main 2010.

Kreidenweis, Helmut/Halfar, Bernd: IT-Report für die Sozialwirtschaft 2010. Wertbeitrag der IT und Markenstärke der Anbieter. Eichstätt 2010.

Kreidenweis, Helmut/Halfar, Bernd: IT-Report für die Sozialwirtschaft 2011. Eichstätt 2011. Kreidenweis, Helmut: Nützliche Technik: Der Computer hilft in der Pflege. In: Altenpflege 5/2009.

Johannes Koukouliatas: Was taugt Fachsoftware für die Sozialwirtschaft? In: Sozialwirtschaft 2/2012.

\section{Gut beraten.}

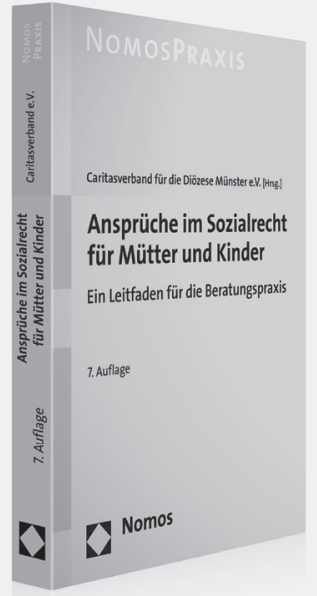

\section{Ansprüche im Sozialrecht für Mütter und Kinder}

Ein Leitfaden für die Beratungspraxis Herausgegeben vom Caritasverband für die Diözese Münster e.V.

Verfasst von:

Rechtsanwältin Birgit Scheibe

7. Auflage 2012, ca. 300 S.,

brosch., ca. 24,- $€$

ISBN 978-3-8329-5969-2

Erscheint ca. April 2012

Auf aktuellem Stand und natürlich unter Berücksichtigung der grundlegenden Änderungen durch die HartzIV-Reform sowie der Änderungen beim Elterngeld und in der Krankenversicherung bietet die Neuauflage des Leitfadens allen Sozialrechtsberatern/-innen systematisch wie praxisorientiert Lösungen zu Fragen der juristischen Beratungspraxis, z.B. zu den Beratungslagen

www.nomos-shop.de/12910

Nomos 